\title{
EFFECT OF VIEW DISTANCE AND MOVEMENT SCALE ON HAPTIC- BASED TELEOPERATION OF INDUSTRIAL ROBOTS IN COMPLEX ENVIRONMENTS
}

\author{
Pholchai Chotiprayanakul $^{1 *}$, Dikai Liu ${ }^{2}$, and Gavin Paul ${ }^{2}$ \\ ${ }^{1}$ Faculty of Engineering, King Mongkut's Institute of Technology, Ladkrabang Chalong-Krung, \\ Bangkok, Thailand \\ ${ }^{2}$ Centre for Autonomous Systems, Faculty of Engineering and Information Technology, \\ University of Technology, Sydney, Australia \\ *Corresponding author (kcpholch@kmitl.ac.th)
}

\begin{abstract}
This paper presents the study on the effect of view distance and movement scale on performance of haptic based teleoperation of a sandblasting robot in complex steel bridge maintenance environments. The operational performance, measured by the Index of Performance (IP), is defined based on the speed and the control accuracy of the manipulator. View distance (i.e. the distance between a display space and an object movement space) and movement scale between hand movement and manipulator movement, which are normally selected empirically, have significant effect the performance. In this paper, an experimental approach is used for determining view distance and movement scale. The sandblasting robotic system is used as an example industrial application in the experiments. Results of the experiments show a range of the view distance and the movement scale that can improve the performance of haptic-based teleoperation of industrial robots in complex environments.
\end{abstract}

Keywords: Human-Robot Interaction, Movement Scale, View Distance, Steel Bridge Maintenance Robot

\section{INTRODUCTION}

Industrial robots have started to be deployed in environments that are complex, unstructured and dynamic changing. Steel bridge maintenance robot is one of the examples [1]. Due to the complexity of steel bridges, human interaction in robot operation is required as fully autonomous operation is only feasible for some applications and environments.

In the steel bridge maintenance robotic system, a robot arm affixed with a sandblasting nozzle is remotely controlled to strip paint or rust from surface of steel bridge structures [1]. The sandblasting robot is expected to operate in complex and unknown (or partially known) environments such as those in construction and steel bridge maintenance, to assist human workers in undertaking hazardous tasks. In manual and semi-automatic operations of this robotic system, a haptic device is used to steer the robot to follow defined blasting paths and provide the kinesthetic feedback to the operator's hand [3][4]. This approach utilises human intelligence and experience combined with the power and accuracy of an industrial robot to address the environment complexity and uncertainty, and handle the big sandblasting force.

Fig. 1a shows the haptic-based operation of an industrial robot in complex environment and Fig. $1 \mathrm{~b}$ shows the sandblasting robotic system for steel bridge maintenance. The sandblasting robotic system consists of a robot arm affixed with sandblasting equipment and placed on a mobile platform. A haptic device with force feedback is used to operate the sandblasting robot. A virtual spring method [2] is used as the interface between the haptic device and the robot arm for guiding the robot to approach target points. 


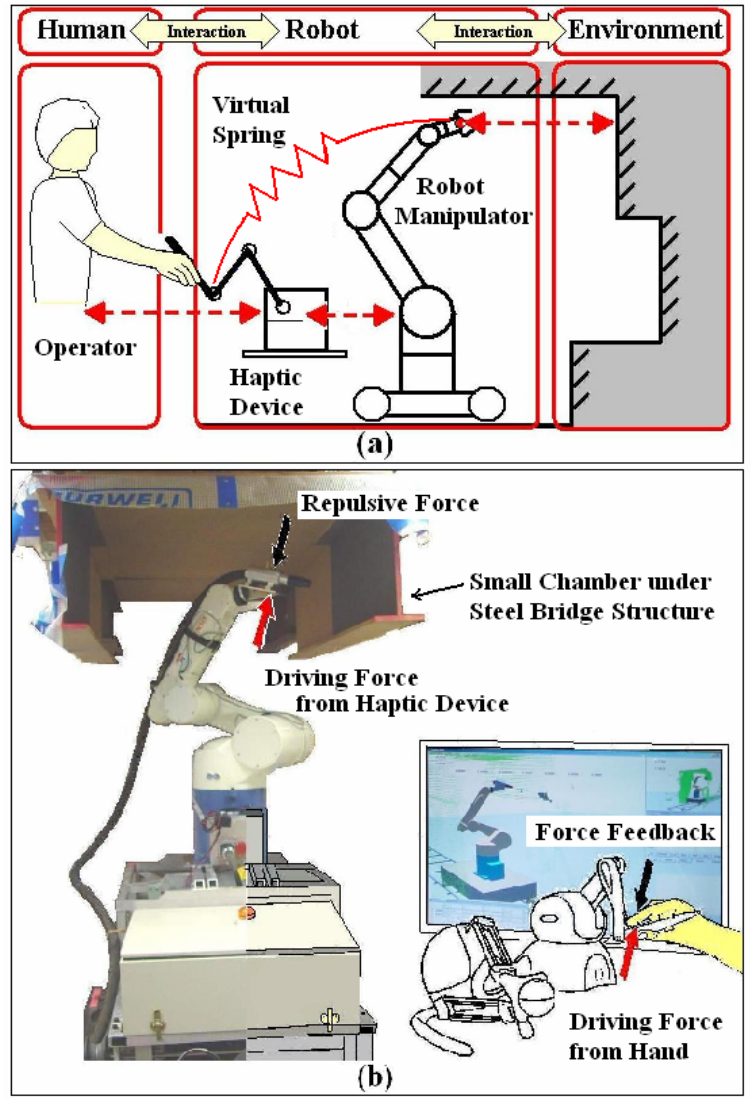

Fig. 1.a) Haptic based operation of an industrial robot in a complex environment and b) Haptic-based sandblasting robotic system [3][5]

When human operator is in the operation loop of a robotic system, human factors will have significant effect on the performance of haptic-based operation. This paper aims to investigate the effect of view distance and movement scale on the operational performance in haptic-based robot operation.

The popular human hand movement models, Fitts's law [8] and Accot-Zhai's Steering law [7][10], have been used to predict movement time and indicate the difficulty of using computer-input devices. Fitts' law is mathematically formulated upon data gathered from experiments using a point-to-point movement testing method. Accot-Zhai's Steering law is now used to evaluate GUI design in computer applications, operating systems and computerconsole games. In human-computer interaction as shown in Fig. 2, the dimension of the task space can be scaled up or down by a control-display (CD) scale $\left(G_{e}\right)$ according to
Casiez's work [9]. $G_{e}$ is used to scale the target size $(\phi)$ to a perceived (or displayed) target size $\left(\phi_{e}\right)$.

$G_{e}=\frac{\phi_{e}}{\phi}$

A control-movement $(\mathrm{CM})$ scale $\left(G_{h}\right)$ is used to represent the relationship between a object movement distance $(s)$ and a hand movement distance $\left(s_{h}\right)$ [7][10].

$G_{h}=\frac{s_{h}}{s}$

In computer operation, the control-display scale and control-movement scale map the task space ( $s$ and $\phi$ ) to the operational space $\left(s_{h}\right.$ and $\left.\phi_{e}\right)$.

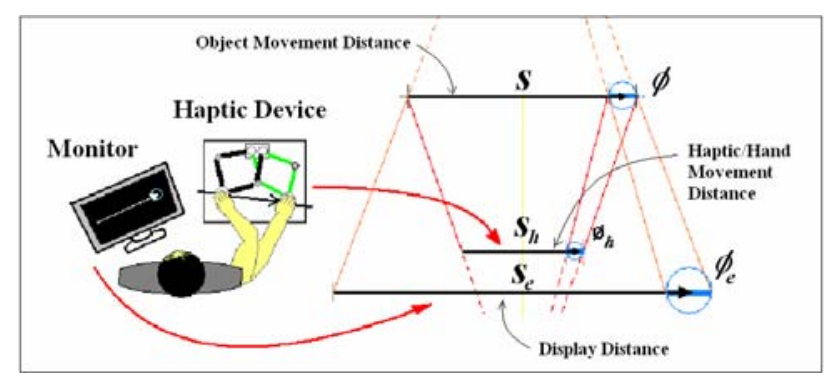

Fig. 2. a) Control-display scale and control-movement scale in human-computer interaction (HCI)

Steering law have been modified by using CM and CD [6][9] and are shown as

$M T=a+b \frac{s}{G_{x} \phi}$

The movement time $(M T)$ is defined by the travelling distance $(s)$, path width $(\phi)$, the control scale $\left(G_{x}=G_{e} / G_{h}\right)$, the start/stop time $(a)$, and the coefficient of movement speed $(b)$. Start/stop time $(a)$ is the time that an operator spends for planning their movement. Munoz [14] presents a way to improve performance in teleoperation through multispace but the control speed and accuracy are not included, only the movement time is measured.

View distance, which is the distance between a display space and an object movement space and related to the location of a surveillance camera, has a significant effect on the performance of haptic-based teleoperation. The view distance is not included in the Steering law. The research presented in [6][9] discussed the control-movement scale 
which amplifies human hand movement, and the controldisplay scale which enlarges or reduces the task space. However, the view distance is not explicitly addressed.

In Steering law, the index of performance also called throughput $(T P=1 / b)[8][11]$ characterises how quickly a pointing task can be completed. $T P$ is the index of overall performance that indicates the movement speed of the operator relative to the index of difficulty [13]. For robotic sandblasting process, operational performance is measured based on speed and accuracy of blasting spot on surface target [12]. The throughput only depends upon the movement speed $(b)$ without considering pointing accuracy or control accuracy. Therefore, the Steering law cannot be directly used to measure the performance of the haptic based teleoperation of industrial robot in complex environment

\section{VIEW DISTANCE AND MOVEMENT SCALE}

\subsection{View Distance}

In real application, a surveillance camera is used to monitor the operation, the target path, and the environments. Size of the target path shown on a monitor is depended on the monitor size and a view distance, which is a distance between a display space and an object movement space. View distance $\left(d_{v}\right)$ is studied as a human factor that affects the operator's performance. With a fixed viewpoint and a fixed distance between the camera and the display space, the relationship between an object movement space and a display space (Fig. 3) is defined as:

$\phi=2 d_{v} \tan \left(\frac{\alpha_{v}}{2}\right)+\phi_{e}$

where $\phi$ is the dimension of the path width on the object movement space and $\phi_{e}$ is the dimension of the path width shown on a display screen space (i.e. a monitor). Thus $d_{v}$ is the distance between an object movement space and a display screen space and $\alpha_{v}$ is the angle of view (i.e. the field of view) of a surveillance camera (Fig. 4). According to the control-display scale [9], $G_{e}$ is now defined as

$$
G_{e}=\frac{\phi_{e}}{2 d_{v} \tan \left(\frac{\alpha_{v}}{2}\right)+\phi_{e}}
$$

By varying $d_{v}$ and $\alpha_{v}$, the projection can be zoomed in or out. For most industry applications, $G_{e}$ is less than 1 because the object space is always bigger than the display screen space. Note that this definition of projection is for a normal camera but the definition of microscope projection must be different.

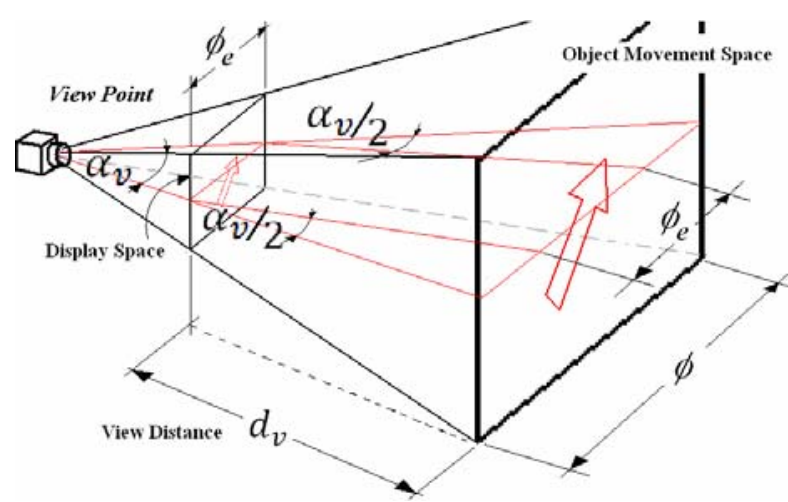

Fig. 3. Object movement space-to-display space projection

\subsection{Movement Scale}

The control-movement scale $\left(G_{h}\right)$ [10] in Equation 2 is used to show the relationship between physical haptic workspace and the object movement space. The size of the physical haptic workspace is enlarged or reduced to the object movement space by this control-movement scale $\left(G_{h}\right)$. That is to say, the hand movement (i.e. the movement of the haptic device handle) is enlarged or reduced by $G_{h}$ times.

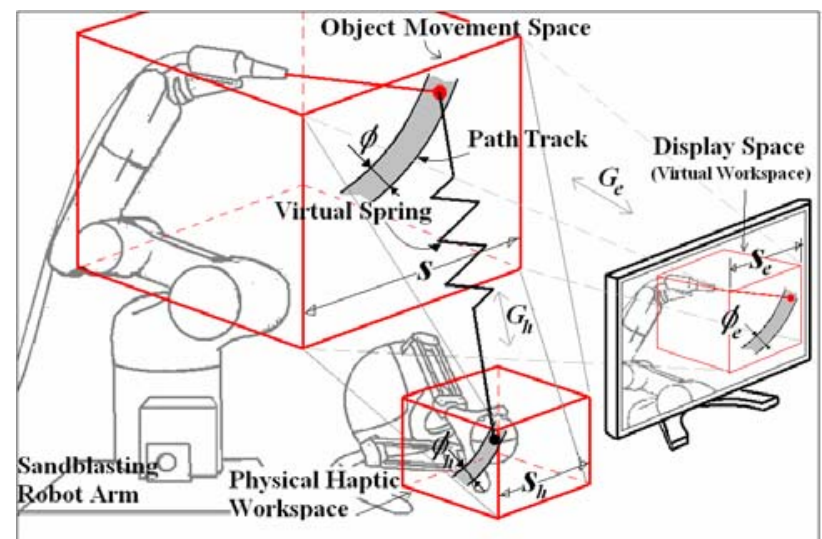

Fig. 4 Control-display scale and control-movement scale in human-robot interaction (HRI); object movement space, display space, and physical haptic workspace 


\section{INDEX OF PERFORMANCE}

In order to investigate the effect of the view distance and the movement scale on the operational performance of the haptic-based teleoperation of the sandblasting robot, a performance measurement needs to be defined. When an operator interacts with a robot manipulator using a haptic device, such as in the application of a sandblasting robotic system, the operator controls the movement of the endeffector of the manipulator by moving the haptic cursor through a haptic device to follow a desired path within a defined path width. The pointing accuracy of the endeffector is defined as the error of position offset (i.e. distance $\boldsymbol{r}_{\boldsymbol{a}}-\boldsymbol{r}_{\boldsymbol{b}}$ ) between the end-effector actual trajectory and the desired trajectory (i.e. the middle line of the desired path) as shown in Fig. 5. The distribution of distance $\boldsymbol{r}_{\boldsymbol{a}}-\boldsymbol{r}_{\boldsymbol{b}}$ is defined as normal distribution. Assume the width of the desired path is $\phi$ and the blasting spot needs to stay within the desired path boundary, the pointing accuracy is related to the times that the blasting spot stays inside the path track boundary. The pointing accuracy $(\varphi)$ can be defined as

$\varphi=\frac{\mathrm{P}\left(\frac{-\sigma}{2}<x<\frac{\sigma}{2}\right)}{\sigma}$

where $P$ is a probability density function and $\sigma$ is the standard deviation of the blasting spot position $\left(P_{e}\right)$ on the task plane. From Fig. 5, the standard deviation $(\sigma)$ of distribution of $\boldsymbol{r}_{\boldsymbol{a}}$ is represented by

$\sigma=\sqrt{\frac{1}{N} \sum_{i=1}^{N}\left(r_{a i}-r_{b}\right)^{2}} ; N$ is sampling number

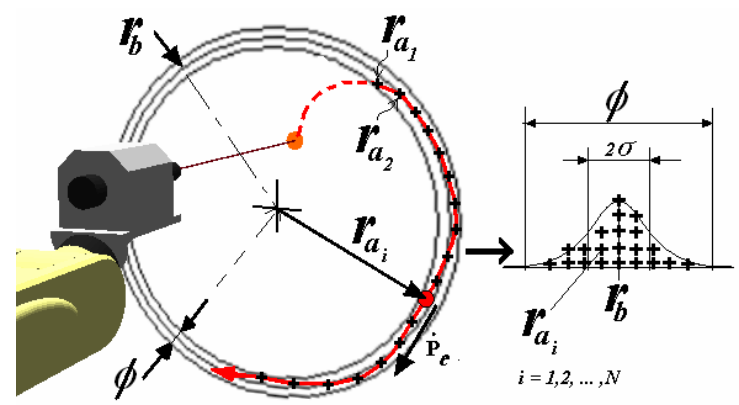

Fig. 5. Error distribution

Thus, the index of performance $(I P)$ used in this paper is defined by the product of the speed and the pointing accuracy of the manipulator end-effector (e.g. the blasting spot in sandblasting operation) when it moves along a target path with a defined boundary:

$I P=\dot{P}_{e} \varphi$

\section{EXPERIMENT}

This experiment is conducted by using the sandblasting robot and implemented with a Novint ${ }^{\circledR}$ haptic device. In this experiment, the view distance is determined in order to maximize performance of operators. Only three joints (Joints 1, 2 and 3) of the robot manipulator are controlled and the other three joints are set to be rigid joints to avoid the redundancy in robot position calculation.

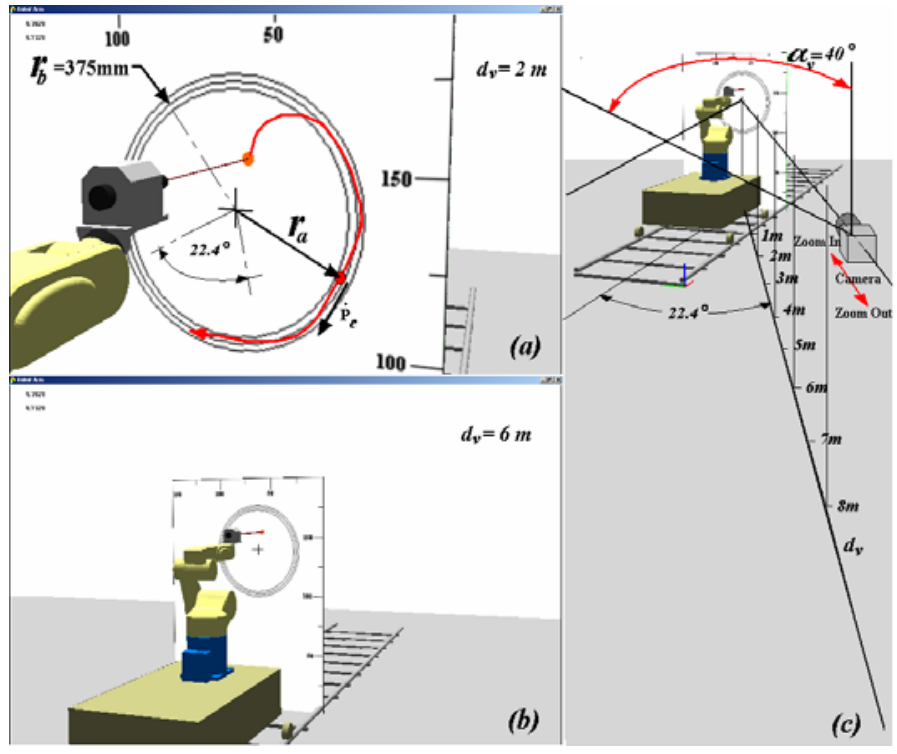

Fig. 6. Setup of the experimental test with different view distances $\left(d_{v}\right)$; (a) Image of view distance at $d_{v}=2 \mathrm{~m}$, (b) Image of view distance at $d_{v}=6 \mathrm{~m}$, and (c) Setup of view distance $\left(d_{v}\right)$

In this experiment, an operator controls the end-effector of the manipulator (defined as the sandblasting spot in the sandblasting robot) using the Novint ${ }^{\circledR}$ haptic device to move within a circular path track for three minutes, as shown in Fig. 6 . The width of the circular path track $(\phi)$ is $40 \mathrm{~mm}$, and the radius of the track $\left(\boldsymbol{r}_{b}\right)$ is $375 \mathrm{~mm}$. The view distance $\left(d_{v}\right)$ is set at $2 \mathrm{~m}, 4 \mathrm{~m}, 6 \mathrm{~m}$, and $8 \mathrm{~m}$ respectively, and the control-display scales $\left(G_{e}\right)$ calculated by Equation 5 from these view distances are $0.185,0.102$, 0.070 , and 0.054 respectively. The angle of view between 
the camera axis and a normal vector of the target plane is set to 22.4 degrees (as shown in detail in Fig. 6). The field of view $\left(\alpha_{v}\right)$ of the surveillance camera is 40 degrees. In this experiment, a 13 inch screen (diagonal) with $1280 \times 800$ resolutions is used for monitoring the operation. The control-movement scale $\left(G_{h}\right)$ is tested at 5 different scales $0.023,0.027,0.045,0.067$, and 0.09 .

\section{RESULTS}

In this experiment, the end-effector of the robot manipulator (the blasting spot) speed $\left(\dot{P}_{e}\right)$ and distance $\left(\boldsymbol{r}_{\boldsymbol{a}}\right)$ are recorded over the test period. The distance $\left(\boldsymbol{r}_{\boldsymbol{a}}\right)$ is then converted to $\sigma$ by Equation 7. $\sigma$ and $\dot{P}_{e}$ are used to calculate the index of performance $(I P)$ with Equation 8. Six participants are involved in this experiment and the experimental results are shown in Table 1. Base upon Table 1, the graphs in Fig. 7 show the index of performance (IP), which Fig. 7a is based on $d_{v}$ while Fig. $7 \mathrm{~b}$ is based on $\mathrm{G}_{\mathrm{e}}$. For the maximum performance, Fig. 7a shows that index of performance (IP) is highest when the view distance is about $4.5 \mathrm{~m}$ and Fig. $7 \mathrm{~b}$ shows that the $I P$ is the highest when $G_{e}$ is about 0.05 .

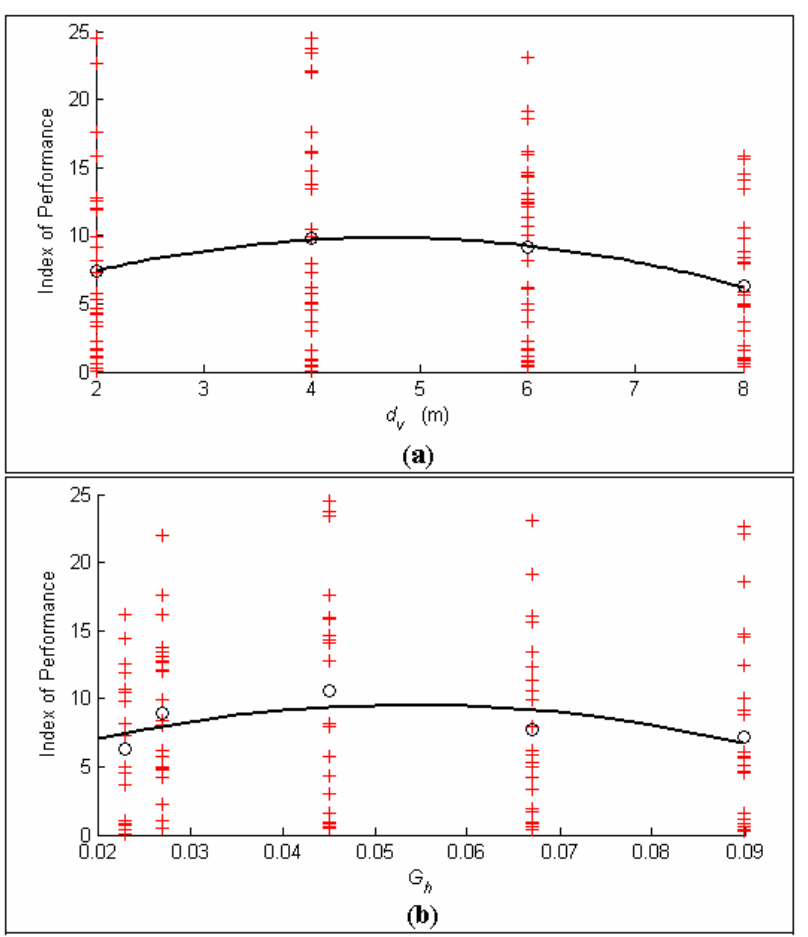

Fig. 7. IPS of 6 participants are shown as red points and the

Average $I P$ of the 6 participants are shown in black lines.
Table 1. Index of performance (IP) of 6 participants

\begin{tabular}{|c|c|c|c|c|c|c|c|}
\hline \multirow{2}{*}{$d_{v}$} & \multirow[b]{2}{*}{ Participant } & \multicolumn{5}{|c|}{$G_{h}$} & \multirow[b]{2}{*}{ Average } \\
\hline & & 0.09 & 0.067 & 0.045 & 0.027 & 0.023 & \\
\hline \multirow{6}{*}{$2 m$} & $\# 1$ & 22.61 & 5.2712 & 4.3163 & 12.738 & 8.1647 & \multirow{6}{*}{7.3832} \\
\hline & \#2 & 0.6343 & 3.3815 & 24.541 & 1.004 & 7.2426 & \\
\hline & \#3 & 0.3149 & 4.2517 & 15.812 & 11.954 & 12.509 & \\
\hline & $\# 4$ & 4.6391 & 9.9075 & 5.7018 & 17.585 & 11.887 & \\
\hline & $\# 5$ & 9.1181 & 0.5544 & 12.789 & 4.2292 & 0.0062 & \\
\hline & \#6 & 1.1085 & 1.6747 & 1.6446 & 2.2386 & 3.6671 & \\
\hline \multirow{6}{*}{$4 m$} & $\# 1$ & 5.0979 & 16.115 & 24.455 & 13.725 & 16.142 & \multirow{6}{*}{9.8107} \\
\hline & $\# 2$ & 5.756 & 6.2144 & 23.748 & 9.8839 & 7.3271 & \\
\hline & \#3 & 14.781 & 0.8174 & 23.398 & 21.982 & 10.516 & \\
\hline & $\# 4$ & 22.071 & 0.9585 & 17.575 & 0.4763 & 0.3899 & \\
\hline & $\# 5$ & 4.5885 & 13.461 & 7.8951 & 5.7895 & 0.0263 & \\
\hline & $\# 6$ & 1.5473 & 7.9665 & 2.9739 & 4.9382 & 3.7055 & \\
\hline \multirow{6}{*}{$6 \mathrm{~m}$} & $\# 1$ & 10.042 & 12.327 & 14.286 & 16.126 & 10.64 & \multirow{6}{*}{9.1206} \\
\hline & $\# 2$ & 0.8721 & 11.352 & 15.937 & 12.072 & 4.5009 & \\
\hline & \#3 & 18.577 & 19.153 & 14.647 & 12.7 & 14.455 & \\
\hline & $\# 4$ & 6.073 & 23.09 & 0.4862 & 13.146 & 0.6767 & \\
\hline & $\# 5$ & 1.1085 & 1.6747 & 1.6446 & 2.2386 & 3.6671 & \\
\hline & $\# 6$ & 12.466 & 0.4148 & 8.1515 & 6.1453 & 4.9467 & \\
\hline \multirow{7}{*}{$8 m$} & $\# 1$ & 14.547 & 10.555 & 15.817 & 13.447 & 9.7949 & \multirow{6}{*}{6.2828} \\
\hline & $\# 2$ & 8.7846 & 15.652 & 0.9419 & 8.1077 & 9.85 & \\
\hline & $\# 3$ & 0.6354 & 4.9804 & 14.052 & 4.913 & 0.8711 & \\
\hline & $\# 4$ & 5.6197 & 5.8586 & 0.8508 & 8.3488 & 0.9955 & \\
\hline & $\# 5$ & 1.5473 & 7.9665 & 2.9739 & 4.9382 & 3.7055 & \\
\hline & $\# 6$ & 0.3880 & 1.96 & 0.5909 & 4.7876 & 5.0044 & \\
\hline & Average & 7.2053 & 7.7316 & 10.634 & 8.8964 & 6.2788 & \\
\hline
\end{tabular}

\section{CONCLUSION}

This paper studied the effect of view distance and movement scale on the operational performance of the haptic-based teleoperation of an industrial robot operating. The sandblasting robot is used in the test as an example application. This paper aims to empirical identify the optimal view distance $\left(d_{v}\right)$ and the optimal controlmovement scale $\left(G_{h}\right)$ that help the operator perform fastest and accurately. The results of experiments show that the 
optimal view distance and the optimal movement scale. However, this suggestion is made based on the sandblasting robotic system which is operated at the specific angle of view (22.4 degree). For other systems and different view angles, similar experiments need to be conducted. The difference in performance of the six participants (as shown in Fig. 7, red points) is mainly caused by individual ability of hand movement, operator's eyesight, and experience with computer operation, haptic device and robot operation.

\section{REFERENCES}

[1] D. K. Liu, G. Dissayanake, P. B. Manamperi, P. A. Brooks, G. Fang, G. Paul, S. Webb, N. Kirchner, P, Chotiprayanakul, N. M. Kwok and T. R. Ren, 2008, 'A Robotic System for Steel Bridge Maintenance: Research Challenges and System Design', paper presented at the 2008 Australasian Conference on Robotics and Automation, 3-5 December 2008, Canberra, Australia.

[2] J. Park and O. Khatib, 2006, 'A Haptic Teleoperation Approach Based on Contact Force Control', International Journal of Robotics Research, vol. 25, no. 56, pp. 575-591.

[3] P. Chotiprayanakul, D. Wang, N. M. Kwok and D.K. Liu, 2008, 'A Haptic Base Human Robot Interaction Approach for Robotic Grit Blasting', Proceedings of the 25th International Symposium on Automation and Robotics in Construction (ISARC'2008), pp. 148-154.

[4] P. Chotiprayanakul and D. K. Liu, 2009, 'Workspace Mapping and Force Control for Small Haptic Device Based Robot Teleoperation', Proceedings of the 2009 IEEE International Conference on Information and Automation (ICIA'2009), pp. 1613-1618.

[5] P. Chotiprayanakul (2011), 'Effective Methods for Human-Robot-Environment Interaction by means of Haptic Robotics', PhD thesis, University of Technology, Sydney, 2011.

[6] J. Accot and S. Zhai, 2001, 'Scale Effects in Steering Law Tasks', Proceedings of the ACM Computer Human Interface Conference, Spatial Interest Group on Computer Human Interaction (SIGCHI), vol. 3, no. 1, pp.
$1-8$.

[7] J. Accot and S. Zhai, 1997, 'Beyond Fitts' Law: Models for Trajectory-Based HCI Tasks', Proceedings of the ACM Computer Human Interface Conference, Spatial Interest Group on Computer Human Interaction (SIGCHI), pp. 295-302.

[8] P. M. Fitt, 1954, 'The Information Capacity of the Human Motor System in Controlling the Amplitude of Movement', Journal of Experimental Psychology, vol. 47, no. 6, pp. 381-391.

[9] G. Casiez, D. Vogel, R. Balakrishnan, and A. Cockburn, 2008, 'The Impact of Control-Display Gain in User Performance in Pointing Tasks', International Journal of Human-Computer Interaction, vol. 23, pp. 215 250

[10] J. Accot and S. Zhai, 2003, 'Refining Fitts' Law Models for Bivariate Pointing', Proceedings of the International Conference on Computer-Human Interaction 2003, vol. 5, no. 1, pp. 193-200.

[11] G. S. Lee and B. Thuraisingham, 2008, 'Differences in Fitts' Law Task Performance Based on Environment Scaling', Haptics: Perception, Devices and Scenarios: 6th International Conference EuroHaptic 2008, Springer-Verlag, Berlin, pp. 295-300.

[12] Mikell P. Groover, 2007, 'Automation, Production Systems, and Computer-Integrated Manufacturing System', Prentice Hall, pp.49-68.

[13] Richard C. Simpson, 2009, 'Using the SpeedAccuracy Operating Characteristic to Visualize Performance with Pointing Device', Journal of The Open Rehabilitation, vol.2, pp. 58-63.

[14] L. M. Munoz and A. Casals, 2009, 'Improving the Human-Robot Interface Through Adaptive Multispace Transformation', IEEE Transactions on Robotics, vol. 25, no. 5 , pp. $1208-1213$. 